\title{
DOCTORS BY THEMSELVES
}

An anthology compiled by Edward F. Griffith, M.R.C.S., L.R.C.P. Pp. xxii + 614, with 64 illustrations. London: Cassell \& Co., Ltd. 1951. 21 s.

The history of medicine is an enormous subject, and the majority of books which deal with it, however thorough in their composition, suffer from a certain dryness of atmosphere which, though almost inevitable, is often a deterrent to many doctors whose interest in their profession does not stop short at the mere technique of their art. In this book we have a fascinating introduction not only to the story of the gradual development of medicine from the earliest times up to the present day but also to the personal character of those who, by their lives and work, have written that story for our delectation and for our example.

Most anthologies, whether of prose or of verse, are stamped with the individuality of their compilers, whose choice does not always correspond with that of the majority of their readers. Dr. Griffith's catholicity of selection is one of the principal charms of this attractive volume, and any reader who is unable to find in its pages an abundance of material to please him and to improve his mind is, in our judgment, a poor and sorry pupil.

Two important features of this work are first the index of sources, and second the biographical notes, both of which are invaluable. The latter are short but adequate, and give the reader a useful lead to further study of the lives of the many illustrious men whose writings are quoted. The references given in the former are a notable addition to the volume as a whole, and the author is to be congratulated on the labour and energy which the preparation of such a list must have involved. .

We regard this publication as a new departure in the literature of the history of medicine. It should be on the bookshelf of every medical man who has any pretence to culture and to a genuine interest in the life of medicine and of doctors. We congratulate both author and publishers and we warmly recommend the book to all readers of good taste.

MaURice Davidson. 